\title{
Degenerate Changhee-Genocchi numbers and polynomials
}

\author{
Byung Moon Kim ${ }^{1}$, Lee-Chae Jang ${ }^{2 *}$, Wonjoo Kim ${ }^{3}$ and Hyuck-In Kwon ${ }^{4}$
}

"Correspondence:
Icjang@konkuk.ac.kr
${ }^{2}$ Graduate School of Education,
Konkuk University, Seoul, 143-701,
Republic of Korea
Full list of author information is
available at the end of the article

available at the end of the article

\begin{abstract}
In this paper, we study some properties of degenerate Changhee-Genocchi numbers and polynomials and give some new identities of these polynomials and numbers which are derived from the generating function. In particular, we provide interesting identities related to the Changhee-Genocchi polynomials of the second kind and Changhee-Genocchi numbers of the second kind.
\end{abstract}

MSC: $05 \mathrm{~A} 10 ; 05 \mathrm{~A} 19 ; 11 \mathrm{~B} 68 ; 11 \mathrm{~S} 80$

Keywords: Changhee numbers; Genocchi numbers; degenerate Changhee-Genocchi numbers

\section{Introduction}

Carlitz first introduced the concept of degenerate numbers and polynomials which are related to Bernoulli and Euler numbers and polynomials (see [1, 2]). After Carlitz introduced the degenerate polynomials, many researchers studied the degenerate polynomials associated with special polynomials in various areas (see [3-20]).

Recently, Kim and Kim gave same new and interesting identities of Changhee numbers and polynomials which are derived from the non-linear differential equation (see [9]). These identities and technical method are very useful for studying some problems which are related to mathematical physics.

As is known, the Genocchi polynomials are defined by the generating function to be

$$
\frac{2 t}{e^{t}+1} e^{x t}=\sum_{n=0}^{\infty} G_{n}(x) \frac{t^{n}}{n !} \quad(\text { see }[1-25]) .
$$

When $x=0, G_{n}=G_{n}(0)$ are called the Genocchi numbers. From (1.1), we note that

$$
G_{0}(x)=0, \quad E_{n}(x)=\frac{G_{n+1}(x)}{n+1} \quad(n \geq 0) \quad(\text { see }[16]) .
$$

Here, $E_{n}(x)$ are ordinary Euler polynomials which are given by the generating function to be

$$
\frac{2}{e^{t}+1} e^{x t}=\sum_{n=0}^{\infty} E_{n}(x) \frac{t^{n}}{n !}
$$


Recently, the Changhee polynomials have been defined by the generating function to be

$$
\frac{2}{t+2}(1+t)^{x}=\sum_{n=0}^{\infty} C h_{n}(x) \frac{t^{n}}{n !} \quad(\text { see }[9,11-17])
$$

When $x=0, C h_{n}=C h_{n}(0)$ are called the Changhee numbers. From (1.3), we note that

$$
\begin{aligned}
\sum_{n=0}^{\infty} C h_{n}(x) \frac{t^{n}}{n !} & =\frac{2}{e^{\log (1+t)}+1} e^{\log (1+t) x} \\
& =\sum_{k=0}^{\infty} E_{k}(x) \frac{1}{k !}(\log (1+t))^{k} \\
& =\sum_{n=0}^{\infty}\left(\sum_{k=0}^{n} E_{k}(x) S_{1}(n, k)\right) \frac{t^{n}}{n !}
\end{aligned}
$$

where $S_{1}(n, k)$ is called the Stirling number of the first kind. From (1.4), we note that

$$
C h_{n}(x)=\sum_{k=0}^{n} E_{k}(x) S_{1}(n, k) \quad(n \geq 0) \quad(\text { see }[9])
$$

As is well known, the Bernoulli numbers of the second kind are defined by the generating function to be

$$
\frac{t}{\log (1+t)}=\sum_{n=0}^{\infty} b_{n} \frac{t^{n}}{n !} \quad(\operatorname{see}[24])
$$

From (1.6), we note that

$$
\left(\frac{t}{\log (1+t)}\right)^{r}(1+t)^{x-1}=\sum_{n=0}^{\infty} B_{n}^{(n-r+1)}(x) \frac{t^{n}}{n !}
$$

When $x=1$ and $r=1$, we get

$$
b_{n}=B_{n}^{(n)}(1) \quad(n \geq 0),
$$

where $B_{n}^{(r)}(x)$ are the higher-order Bernoulli polynomials which are defined by the generating function to be

$$
\left(\frac{t}{e^{t}-1}\right)^{r} e^{x t}=\sum_{n=0}^{\infty} B_{n}^{(r)}(x) \frac{t^{n}}{n !} \quad(\text { see }[6,7,11])
$$

In [17], Changhee-Genocchi polynomials are defined by the generating function to be

$$
\frac{2 \log (1+t)}{2+t}(1+t)^{x}=\sum_{n=0}^{\infty} C G_{n}(x) \frac{t^{n}}{n !} .
$$


From (1.8), we note that

$$
\begin{aligned}
\frac{2 \log (1+t)}{2+t}(1+t)^{x} & =\frac{2 \log (1+t)}{e^{\log (1+t)}+1} e^{x \log (1+t)} \\
& =\sum_{k=0}^{\infty} G_{k}(x) \frac{1}{k !}(\log (1+t))^{k} \\
& =\sum_{n=0}^{\infty}\left(\sum_{k=0}^{n} G_{k}(x) S_{1}(n, k)\right) \frac{t^{n}}{n !} .
\end{aligned}
$$

By (1.8) and (1.9), we get

$$
C G_{n}(x)=\sum_{k=0}^{n} G_{k}(x) S_{1}(n, k) \quad(\text { see }[17,18])
$$

Now, we consider the modified Changhee-Genocchi polynomials which are given by

$$
\frac{2 t}{2+t}(1+t)^{x}=\sum_{n=0}^{\infty} C G_{n}^{*}(x) \frac{t^{n}}{n !}
$$

Now, we observe that

$$
\begin{aligned}
\frac{2 t}{2+t}(1+t)^{x} & =\frac{2 t}{e^{\log (1+t)}+1} e^{x \log (1+t)} \\
& =t \sum_{k=0}^{\infty} E_{k}(x) \frac{1}{k !}(\log (1+t))^{k} \\
& =t \sum_{n=0}^{\infty}\left(\sum_{k=0}^{n} E_{k}(x) S_{1}(n, k)\right) \frac{t^{n}}{n !}
\end{aligned}
$$

From (1.11), it is easy to show that $C G_{0}^{*}(x)=0$. Thus, by (1.11) and (1.12), we get

$$
\frac{C G_{n+1}^{*}(x)}{n+1}=\sum_{k=0}^{n} E_{k}(x) S_{1}(n, k) \quad(n \geq 0) .
$$

As is known, the degenerate Euler polynomials were defined by Carlitz $[1,2]$ to be

$$
\frac{2}{(1+\lambda t)^{\frac{1}{\lambda}}+1}(1+\lambda t)^{\frac{x}{\lambda}}=\sum_{n=0}^{\infty} \mathcal{E}_{n, \lambda}(x) \frac{t^{n}}{n !}
$$

Now, we consider the following degenerate Genocchi polynomials which are derived from (1.14):

$$
\frac{2 t}{(1+\lambda t)^{\frac{1}{\lambda}}+1}(1+\lambda t)^{\frac{x}{\lambda}}=\sum_{n=0}^{\infty} G_{n, \lambda}(x) \frac{t^{n}}{n !} .
$$


Thus, by (1.15), we get

$$
\begin{aligned}
\sum_{n=0}^{\infty} \lim _{\lambda \rightarrow 0} G_{n, \lambda}(x) \frac{t^{n}}{n !} & =\lim _{\lambda \rightarrow 0} \frac{2 t}{(1+\lambda t)^{\frac{1}{\lambda}}+1}(1+\lambda t)^{\frac{x}{\lambda}} \\
& =\frac{2 t}{e^{t}+1} e^{x t}=\sum_{n=0}^{\infty} G_{n}(x) \frac{t^{n}}{n !} .
\end{aligned}
$$

From (1.15) and (1.16)

$$
\lim _{\lambda \rightarrow 0} G_{n, \lambda}(x)=G_{n}(x) \quad(n \geq 0) .
$$

It is not difficult to show that

$$
\begin{aligned}
\frac{2}{(1+\lambda t)^{\frac{1}{\lambda}}+1}(1+\lambda t)^{\frac{x}{\lambda}} & =\frac{1}{t} \frac{2 t}{(1+\lambda t)^{\frac{1}{\lambda}}+1}(1+\lambda t)^{\frac{x}{\lambda}} \\
& =\frac{1}{t} \sum_{n=0}^{\infty} G_{n, \lambda}(x) \frac{t^{n}}{n !} \\
& =\frac{1}{t} \sum_{n=1}^{\infty} G_{n, \lambda}(x) \frac{t^{n}}{n !} \\
& =\sum_{n=0}^{\infty} \frac{G_{n+1, \lambda}(x)}{n+1} \frac{t^{n}}{n !}
\end{aligned}
$$

Therefore, by (1.14) and (1.18), for $n \geq 0$, we get

$$
\mathcal{E}_{n, \lambda}(x)=\frac{1}{n+1} G_{n+1, \lambda}(x) .
$$

When $x=0, G_{n, \lambda}=G_{n, \lambda}(0)$ are called the degenerate Genocchi numbers. From (1.15), we have

$$
\begin{aligned}
2 t & =\left(\sum_{l=0}^{\infty} G_{l, \lambda} \frac{t^{l}}{l !}\right)\left(1+(1+\lambda t)^{\frac{1}{\lambda}}\right) \\
& =\sum_{n=0}^{\infty} G_{n, \lambda} \frac{t^{n}}{n !}+\left(\sum_{l=0}^{\infty} G_{l, \lambda} \frac{t^{l}}{l !}\right)\left(\sum_{m=0}^{\infty}(1 \mid \lambda)_{m} \frac{t^{m}}{m !}\right) \\
& =\sum_{n=0}^{\infty}\left\{G_{n, \lambda}+\sum_{l=0}^{n}\left(\begin{array}{l}
n \\
l
\end{array}\right) G_{l, \lambda}(1 \mid \lambda)_{n-l}\right\} \frac{t^{n}}{n !},
\end{aligned}
$$

where $(x \mid \lambda)_{n}=x(x-\lambda) \cdots(x-(n-1) \lambda)(n \geq 1),(x \mid \lambda)_{0}=1$.

By comparing the coefficients on the both sides of (1.19), we have

$$
G_{n, \lambda}+\sum_{l=0}^{n}\left(\begin{array}{l}
n \\
l
\end{array}\right) G_{l, \lambda}(1 \mid \lambda)_{n-l}= \begin{cases}0 & \text { if } n=0 \\
2 \delta_{n, 1} & \text { if } n=1 \\
0 & \text { if } n>1\end{cases}
$$

Note that $G_{0, \lambda}=0$. 
In [17], the degenerate Changhee-Genocchi polynomials are defined by the generating function to be

$$
\frac{2 \lambda \log \left(1+\frac{1}{\lambda} \log (1+\lambda t)\right)}{2 \lambda+\log (1+\lambda t)}\left(1+\lambda^{-1} \log (1+\lambda t)\right)^{x}=\sum_{n=0}^{\infty} C G_{n, \lambda}(x) \frac{t^{n}}{n !} .
$$

From (1.8) and (1.20), we note that

$$
\lim _{\lambda \rightarrow 0} C G_{n, \lambda}(x)=C G_{n}(x) \quad(n \geq 0) \quad(\text { see }[17]) .
$$

The modified degenerate Changhee-Genocchi polynomials are considered by the generating function to be

$$
\frac{2 t \lambda}{2 \lambda+\log (1+\lambda t)}\left(1+\lambda^{-1} \log (1+\lambda t)\right)^{x}=\sum_{n=0}^{\infty} C G_{n, \lambda}^{*}(x) \frac{t^{n}}{n !}
$$

Note that $\lim _{\lambda \rightarrow 0} C G_{n, \lambda}^{*}(x)=C G_{n}^{*}(x)(n \geq 0)$.

As is known, the degenerate Changhee polynomials are given by

$$
\frac{2}{2+\lambda^{-1} \log (1+\lambda t)}\left(1+\lambda^{-1} \log (1+\lambda t)\right)^{x}=\sum_{n=0}^{\infty} C h_{n, \lambda}(x) \frac{t^{n}}{n !} \quad(\text { see }[8]) \text {. }
$$

From (1.22) and (1.23), we have

$$
C h_{n, \lambda}(x)=\frac{1}{n+1} C G_{n+1, \lambda}^{*}(x) \quad(n \geq 0) .
$$

When $x=0, C G_{n, \lambda}^{*}=C G_{n, \lambda}^{*}(0)$ are called the modified degenerate Changhee-Genocchi numbers. From (1.22), for $x=0$, we note that

$$
\begin{aligned}
2 t & =\left(2+\lambda^{-1} \log (1+\lambda t)\right)\left(\sum_{l=0}^{\infty} C G_{l, \lambda}^{*} \frac{t^{l}}{l !}\right) \\
& =\left(2+\sum_{k=1}^{\infty} \frac{(-1)^{k-1} \lambda^{k-1}}{k} t^{k}\right)\left(\sum_{l=0}^{\infty} C G_{l, \lambda}^{*} \frac{t^{l}}{l !}\right) \\
& =\sum_{n=1}^{\infty}\left(2 C G_{n, \lambda}^{*}+\sum_{k=1}^{n}\left(\begin{array}{l}
n \\
k
\end{array}\right)(k-1) !(-1)^{k-1} \lambda^{k-1} C G_{n-k, \lambda}^{*}\right) \frac{t^{n}}{n !} .
\end{aligned}
$$

Comparing the coefficients on the both sides of (1.25), we have

$$
2 C G_{n, \lambda}^{*}+\sum_{k=1}^{n}\left(\begin{array}{l}
n \\
k
\end{array}\right)(k-1) !(-1)^{k-1} \lambda^{k-1} C G_{n-k, \lambda}^{*}=2 \delta_{n, 1},
$$

where $\delta_{n, k}$ is the Kronecker delta symbol. In the viewpoint of (1.8), we consider the following partially degenerate Changhee-Genocchi polynomials which are derived from (1.20):

$$
\frac{2 \lambda \log (1+t)}{2 \lambda+\log (1+\lambda t)}\left(1+\lambda^{-1} \log (1+\lambda t)\right)^{x}=\sum_{n=0}^{\infty} P C G_{n, \lambda}(x) \frac{t^{n}}{n !} .
$$


When $x=0, P C G_{n, \lambda}=P C G_{n, \lambda}(0)$ are called the partially degenerate Changhee-Genocchi numbers. Note that $\lim _{\lambda \rightarrow 0} P C G_{n, \lambda}(x)=C G_{n}(x)(n \geq 0)$.

From (1.27), for $x=0$, we note that

$$
\begin{aligned}
2 \log (1+t) & =\left(2+\lambda^{-1} \log (1+\lambda t)\right)\left(\sum_{l=0}^{\infty} P C G_{l, \lambda} \frac{t^{l}}{l !}\right) \\
& =\sum_{n=1}^{\infty}\left(2 P C G_{n, \lambda}+\sum_{k=1}^{n}(-1)^{k-1} \lambda^{k-1}(k-1) !\left(\begin{array}{l}
n \\
k
\end{array}\right) P C G_{n-k, \lambda}\right) \frac{t^{n}}{n !} .
\end{aligned}
$$

Thus, by (1.28), we get

$$
2 P C G_{n, \lambda}+\sum_{k=1}^{n}(-1)^{k-1} \lambda^{k-1}(k-1) !\left(\begin{array}{l}
n \\
k
\end{array}\right) P C G_{n-k, \lambda}=2(-1)^{n-1}(n-1) !
$$

where $n=1,2,3, \ldots$

In this paper, we study the degenerate Changhee-Genocchi polynomials and numbers of the second kind which are different from previous degenerate Changhee-Genocchi polynomials and numbers. In addition, we give some new identities from our numbers and polynomials.

\section{Changhee-Genocchi numbers and polynomials of the second kind}

First, we consider the Changhee-Genocchi polynomials of the second kind which are given by the generating function to be

$$
\frac{2 \log (1+t)}{(1+\lambda \log (1+t))^{\frac{1}{\lambda}}+1}(1+\lambda \log (1+t))^{\frac{x}{\lambda}}=\sum_{n=0}^{\infty} J_{n, \lambda}(x) \frac{t^{n}}{n !} .
$$

Thus, by (1.8) and (2.1), we get

$$
\sum_{n=0}^{\infty} \lim _{\lambda \rightarrow 0} J_{n, \lambda}(x) \frac{t^{n}}{n !}=\frac{2 \log (1+t)}{2+t}(1+t)^{x}=\sum_{n=0}^{\infty} C G_{n}(x) \frac{t^{n}}{n !} .
$$

By comparing the coefficient on the both sides, we get

$$
C G_{n}(x)=\lim _{\lambda \rightarrow 0} J_{n, \lambda}(x) \quad(n \geq 0)
$$

When $x=0, J_{n, \lambda}=J_{n, \lambda}(0)$ are called the Changhee-Genocchi numbers of the second kind.

From (2.1) and (2.3), we note that

$$
\begin{gathered}
\frac{2 \log (1+t)}{(1+\lambda \log (1+t))^{\frac{1}{\lambda}}+1}(1+\lambda \log (1+t))^{\frac{x}{\lambda}} \\
=(\log (1+t))\left(\sum_{l=0}^{\infty} \mathcal{E}_{l, \lambda}(x) \frac{1}{l !}(\log (1+t))^{l}\right) \\
=(\log (1+t))\left(\sum_{k=0}^{\infty}\left(\sum_{l=0}^{k} \mathcal{E}_{l, \lambda}(x) S_{1}(k, l)\right) \frac{t^{k}}{k !}\right)
\end{gathered}
$$




$$
\begin{aligned}
& =\left(\sum_{m=1}^{\infty} \frac{(-1)^{m-1}}{m} t^{m}\right)\left(\sum_{k=0}^{\infty}\left(\sum_{l=0}^{k} \mathcal{E}_{l, \lambda}(x) S_{1}(k, l)\right) \frac{t^{k}}{k !}\right) \\
& =\sum_{n=1}^{\infty}\left(\sum_{k=0}^{n-1} \sum_{l=0}^{k} \frac{(-1)^{n-k-1} n ! \mathcal{E}_{l, \lambda}(x)}{(n-k) k !} S_{1}(k, l)\right) \frac{t^{n}}{n !}
\end{aligned}
$$

and

$$
\begin{aligned}
\frac{2 \log (1+t)}{(1+\lambda \log (1+t))^{\frac{1}{\lambda}}+1}(1+\lambda \log (1+t))^{\frac{x}{\lambda}} \\
=\sum_{l=0}^{\infty} \mathcal{E}_{l, \lambda}(x) \frac{1}{l !}(\log (1+t))^{l+1} \\
=\sum_{l=0}^{\infty} \mathcal{E}_{l, \lambda}(x)(l+1) \frac{1}{(l+1) !}(\log (1+t))^{l+1} \\
=\sum_{l=0}^{\infty} \mathcal{E}_{l, \lambda}(x)(l+1) \sum_{n=l+1}^{\infty} S_{1}(n, l+1) \frac{t^{n}}{n !} \\
=\sum_{n=1}^{\infty}\left(\sum_{l=0}^{n-1} \mathcal{E}_{l, \lambda}(x)(l+1) S_{1}(n, l+1)\right) \frac{t^{n}}{n !}
\end{aligned}
$$

where $S_{1}(n, k)$ is the Stirling number of the first kind. By (2.1), (2.4) and (2.5), we obtain the following theorem.

Theorem 2.1 For $n \geq 1$, we have

$$
J_{n, \lambda}(x)=\sum_{k=0}^{n-1} \sum_{l=0}^{k} \frac{(-1)^{n-k-1} n !}{(n-k) k !} \mathcal{E}_{l, \lambda}(x) S_{1}(k, l)
$$

and

$$
J_{n, \lambda}(x)=\sum_{l=0}^{n-1} \mathcal{E}_{l, \lambda}(x)(l+1) S_{1}(n, l+1) .
$$

By replacing $t$ by $e^{t}-1$ in (2.1), we get

$$
\begin{aligned}
\frac{2 t}{(1+\lambda t)^{\frac{1}{\lambda}}+1}(1+\lambda t)^{\frac{x}{\lambda}} & =\sum_{k=0}^{\infty} J_{k, \lambda}(x) \frac{1}{k !}\left(e^{t}-1\right)^{k} \\
& =\sum_{k=0}^{\infty} J_{k, \lambda}(x) \sum_{n=k}^{\infty} S_{2}(n, k) \frac{t^{n}}{n !} \\
& =\sum_{n=0}^{\infty}\left(\sum_{k=0}^{n} J_{k, \lambda}(x) S_{2}(n, k)\right) \frac{t^{n}}{n !},
\end{aligned}
$$

where $S_{2}(n, k)$ is the Stirling number of the second kind. Therefore, by (1.15) and (2.6), we obtain the following theorem. 
Theorem 2.2 For $n \geq 0$, we have

$$
G_{n, \lambda}(x)=\sum_{k=0}^{n} J_{k, \lambda}(x) S_{2}(n, k)
$$

From (2.1), we have

$$
\begin{aligned}
2 \log (1+t) & =\left(\sum_{k=0}^{\infty} J_{k, \lambda} \frac{t^{k}}{k !}\right)\left((1+\lambda \log (1+t))^{\frac{1}{\lambda}}+1\right) \\
& =\sum_{n=1}^{\infty} J_{n, \lambda} \frac{t^{n}}{n !}+\left(\sum_{n=1}^{\infty} J_{n, \lambda} \frac{t^{n}}{n !}\right)\left(\sum_{l=0}^{\infty}(1 \mid \lambda)_{l} \frac{1}{l !}(\log (1+t))^{l}\right) \\
& =\sum_{n=1}^{\infty} J_{n, \lambda} \frac{t^{n}}{n !}+\left(\sum_{n=1}^{\infty} J_{n, \lambda} \frac{t^{n}}{n !}\right)\left(\sum_{m=0}^{\infty}\left(\sum_{l=0}^{m}(1 \mid \lambda)_{l} S_{1}(m, l)\right) \frac{t^{m}}{m !}\right) \\
& =\sum_{n=1}^{\infty} J_{n, \lambda} \frac{t^{n}}{n !}+\sum_{n=1}^{\infty}\left(\sum_{m=0}^{n-1} \sum_{l=0}^{m}\left(\begin{array}{l}
n \\
m
\end{array}\right)(1 \mid \lambda)_{l} S_{1}(m, l) J_{n-m, \lambda}\right) \frac{t^{n}}{n !} \\
& =\sum_{n=1}^{\infty}\left\{J_{n, \lambda}+\sum_{m=0}^{n-1} \sum_{l=0}^{m}\left(\begin{array}{c}
n \\
m
\end{array}\right)(1 \mid \lambda)_{l} S_{1}(m, l) J_{n-m, \lambda}\right\} \frac{t^{n}}{n !} .
\end{aligned}
$$

It is easy to show that

$$
2 \log (1+t)=2 \sum_{n=1}^{\infty} \frac{(-1)^{n-1}}{n} t^{n}=2 \sum_{n=1}^{\infty}(-1)^{n-1}(n-1) ! \frac{t^{n}}{n !}
$$

Therefore, by (2.7) and (2.8), we obtain the following theorem.

Theorem 2.3 For $n \geq 1$, we have

$$
J_{n, \lambda}+\sum_{m=0}^{n-1} \sum_{l=0}^{m}\left(\begin{array}{l}
n \\
m
\end{array}\right)(1 \mid \lambda)_{l} S_{1}(m, l) J_{n-m, \lambda}=2(-1)^{n-1}(n-1) !
$$

The degenerate Changhee polynomials of the second kind are also defined by the generating function to be

$$
\frac{2}{(1+\lambda \log (1+t))^{\frac{1}{\lambda}}+1}(1+\lambda \log (1+t))^{\frac{x}{\lambda}}=\sum_{n=0}^{\infty} C_{n, \lambda}(x) \frac{t^{n}}{n !} \quad(\text { see [8] }) .
$$

Now, we observe that

$$
\begin{aligned}
& \frac{2}{(1+\lambda \log (1+t))^{\frac{1}{\lambda}}+1}(1+\lambda \log (1+t))^{\frac{x}{\lambda}} \\
& =\frac{1}{t}\left(\frac{t}{\log (1+t)}\right)\left(\frac{2 \log (1+t)}{(1+\lambda \log (1+t))^{\frac{1}{\lambda}}+1}(1+\lambda \log (1+t))^{\frac{x}{\lambda}}\right) \\
& =\frac{1}{t}\left(\sum_{l=0}^{\infty} b_{l} \frac{t^{l}}{l !}\right)\left(\sum_{m=1}^{\infty} J_{m, \lambda}(x) \frac{t^{m}}{m !}\right)
\end{aligned}
$$




$$
\begin{aligned}
& =\left(\sum_{l=0}^{\infty} b_{l} \frac{t^{l}}{l !}\right)\left(\sum_{m=0}^{\infty} \frac{J_{m+1, \lambda}(x)}{m+1} \frac{t^{m}}{m !}\right) \\
& =\sum_{n=0}^{\infty}\left(\sum_{m=0}^{n}\left(\begin{array}{c}
n \\
m
\end{array}\right) \frac{J_{m+1, \lambda}(x)}{m+1} b_{n-m}\right) \frac{t^{n}}{n !} .
\end{aligned}
$$

By comparing the coefficients on the both sides of (2.9) and (2.10), we obtain the following theorem.

Theorem 2.4 For $n \geq 0$, we have

$$
C_{n, \lambda}(x)=\sum_{m=0}^{n}\left(\begin{array}{c}
n \\
m
\end{array}\right) \frac{J_{m+1, \lambda}(x)}{m+1} b_{n-m}
$$

From (2.1), we easily note that

$$
\begin{aligned}
\sum_{n=0}^{\infty} J_{n, \lambda}(x) \frac{t^{n}}{n !} & =\frac{2 \log (1+t)}{(1+\lambda \log (1+t))^{\frac{1}{\lambda}}+1}(1+\lambda \log (1+t))^{\frac{x}{\lambda}} \\
& =\left(\sum_{l=0}^{\infty} J_{l, \lambda} \frac{t^{l}}{l !}\right)\left(\sum_{m=0}^{\infty}\left(\frac{x}{\lambda}\right)_{m} \lambda^{m} \frac{1}{m !}(\log (1+t))^{m}\right) \\
& =\left(\sum_{m=0}^{\infty}(x \mid \lambda)_{m} \sum_{k=m}^{\infty} S_{1}(k, m) \frac{t^{k}}{k !}\right)\left(\sum_{l=0}^{\infty} J_{l, \lambda} \frac{t^{l}}{l !}\right) \\
& =\left(\sum_{k=0}^{\infty}\left(\sum_{m=0}^{k}(x \mid \lambda)_{m} S_{1}(k, m)\right) \frac{t^{k}}{k !}\right)\left(\sum_{l=0}^{\infty} J_{l, \lambda} \frac{t^{l}}{l !}\right) \\
& =\sum_{n=0}^{\infty}\left(\sum_{k=0}^{n} \sum_{m=0}^{k}\left(\begin{array}{l}
n \\
k
\end{array}\right)(x \mid \lambda)_{m} S_{1}(k, m) J_{n-k, \lambda}\right) \frac{t^{n}}{n !} .
\end{aligned}
$$

Therefore, by comparing the coefficients on the both sides of (2.11), we obtain the following theorem.

Theorem 2.5 For $n \geq 0$, we have

$$
J_{n, \lambda}(x)=\sum_{k=0}^{n} \sum_{m=0}^{k}\left(\begin{array}{l}
n \\
k
\end{array}\right)(x \mid \lambda)_{m} S_{1}(k, m) J_{n-k, \lambda} .
$$

\section{Conclusions}

Kwon et al. [17] introduced the degenerate Changhee-Genocchi polynomials and numbers. In this study, we defined the modified degenerate Changhee-Genocchi polynomials and numbers (see (1.22)) and obtained an interesting identity (1.26) of the modified degenerate Changhee-Genocchi numbers. Secondly, we defined the partially degenerate Changhee-Genocchi polynomials and numbers (see (1.27)). We obtained a useful identity (1.29) of the partially degenerate Changhee-Genocchi numbers (1.29). Finally, we defined the Changhee-Genocchi polynomials of the second kind (see (2.1)). We provided useful identities related to the Changhee-Genocchi polynomials of the second kind and the degenerate Euler polynomials (see Theorem 2.1). Furthermore, we obtained some interesting 
identities of the Changhee-Genocchi polynomials of the second kind (see Theorem 2.2, Theorem 2.3, Theorem 2.4 and Theorem 2.5).

\author{
Acknowledgements \\ The authors would like to express their gratitude for the valuable comments and suggestions of referees.
}

\title{
Competing interests
}

The authors declare that they have no competing interests.

\section{Authors' contributions}

All authors contributed equally to this work. All authors read and approved the final manuscript.

\section{Author details}

${ }^{1}$ Department of Mechanical System Engineering, Dongguk University, Gyeongju, 780-714, Republic of Korea. ${ }^{2}$ Graduate School of Education, Konkuk University, Seoul, 143-701, Republic of Korea. ${ }^{3}$ Department of Applied Mathematics, Kyunghee University, Seoul, 02447, Republic of Korea. ${ }^{4}$ Department of Mathematics, Kwangwoon University, Seoul, 139-701, Republic of Korea.

\section{Publisher's Note}

Springer Nature remains neutral with regard to jurisdictional claims in published maps and institutional affiliations.

Received: 1 October 2017 Accepted: 17 November 2017 Published online: 28 November 2017

\section{References}

1. Carlitz, L: Degenerate Stirling, Bernoulli and Eulerian numbers. Util. Math. 15, 51-88 (1979)

2. Carlitz, L: A degenerate Staudt-Clausen theorem. Arch. Math. (Basel) 7, 28-33 (1956)

3. Kim, B-M, Jeong, J, Rim, S-H: Some explicit identities on Changhee-Genocchi polynomials and numbers. Adv. Differ. Equ. 2016, Article ID 202 (2016)

4. Dolgy, DV, Kim, DS, Kim, T, Mansour, T: Degenerate poly-Cauchy polynomials. Appl. Math. Comput. 269, 637-646 (2015)

5. Kim, DS, Kim, T: Some identities of degenerate Euler polynomials arising from $p$-adic fermionic integrals on $\mathbb{Z}_{p}$. Integral Transforms Spec. Funct. 26, 198-204 (2015)

6. Kim, DS, Kim, T: A note on degenerate poly-Bernoulli numbers and polynomials. Adv. Differ. Equ. 2015, Article ID 258 (2015)

7. Kim, DS, Kim, T, Dolgy, DV: A note on degenerate Bernoulli numbers and polynomials associated with $p$-adic invariant integral on $\mathbb{Z}_{p}$. Appl. Math. Comput. 259, 198-204 (2015)

8. Kim, T, Kim, DS: Degenerate Changhee numbers and polynomials of the second kind. arXiv:1707.09721

9. Kim, T, Kim, DS: A note on nonlinear Changhee differential equations. Russ. J. Math. Phys. 23(1), 88-92 (2016)

10. Kim, T: A note on degenerate Stirling polynomials of the second kind. Proc. Jangjeon Math. Soc. 20(3), $319-331$ (2017)

11. Kim, T, Seo, JJ: A note on partially degenerate Bernoulli numbers and polynomials. J. Math. Anal. 6(5), 1-6 (2015)

12. Kim, T: On the degenerate Cauchy numbers and polynomials. Proc. Jangjeon Math. Soc. 18(3), 307-312 (2015)

13. Kim, T: $\lambda$-analogue of Stirling numbers of the first kind. Adv. Stud. Contemp. Math. (Kyungshang) 27(3), 423-429 (2017)

14. Kim, T, Seo, JJ: On the generalized degenerate Euler numbers and polynomials. Proc. Jangjeon Math. Soc. 18(4), 537-546 (2015)

15. Kim, T, Kim, DS: Identities involving degenerate Euler numbers and polynomials arising from non-linear differential equations. J. Nonlinear Sci. Appl. 9(5), 2086-2098 (2016)

16. Kim, T: On the multiple $q$-Genocchi and Euler numbers. Russ. J. Math. Phys. 15(4), 481-486 (2008)

17. Kwon, H-I, Kim, T, Park, JW: A note on degenerate Changhee-Genocchi polynomials and numbers. Glob. J. Pure Appl. Math. 12(5), 4057-4064 (2016)

18. Lee, JG, Jang, LC, Seo, JJ, Choi, SK, Kwon, HI: On Appell-type Changhee polynomials and numbers. Adv. Differ. Equ. 2016, Article ID 160 (2016)

19. Park, J-W, Jang, G-W, Kwon, J: The $\lambda$-analogue degenerate Changhee polynomials and numbers. Glob. J. Pure Appl. Math. 13(3), 893-900 (2017)

20. Park, KH, Kim, Y-H: On some arithmetical properties of the Genocchi numbers and polynomials. Adv. Differ. Equ. 2008, Article ID 195049 (2008)

21. Cenkci, M, Howard, FT: Notes on degenerate numbers. Discrete Math. 307, 2359-2375 (2007)

22. Qi, F, Jang, LC, Kwon, H-l: Some new and explicit identities related with the Appell-type degenerate $q$-Changhee polynomials. Adv. Differ. Equ. 2016, Article ID 180 (2016)

23. Lim, D: Some identities of degenerate Genocchi polynomials. Bull. Korean Math. Soc. 53(2), 569-579 (2016)

24. Roman, S: The Umbral Calculus, p. 116. Dover, New York (2005)

25. Simsek, Y: Identities on the Changhee numbers and Apostol-type Daehee polynomials. Adv. Stud. Contemp. Math. (Kyungshang) 27(2), 199-212(2017) 